\section{Aerosolized colistin in the treatment of multiresistant Pseudomonas aeruginosa nosocomial pneumonia}

\author{
BOUBAKER CHARRA - ABDELHAMID HACHIMI • \\ ABDELLATIF BENSLAMA • SAID MOTAOUAKKIL
}

SAID MOTAOUAKKIL $(\bowtie) \bullet$ BOUBAKER CHARRA • ABDELHAMID HACHIMI • ABDELLATIF BENSLAMA Service de Réanimation Médicale CHU Ibn Rochd 1, Rue des Hôpitaux

Quartier des Hôpitaux

Casablanca 20 100, Morocco Phone: 0021222228817

E-mail: smotaouakkil@yahoo.fr reamedcasa@gmail.com

\begin{abstract}
Introduction. Multiresistant Pseudomonas aeruginosa (MRPA) nosocomial pneumonia is a significant cause of mortality and morbidity in the ICU. We report our experience with aerosolized colistin in the treatment of MRPA nosocomial pneumonia. Patients and methods. It is a prospective, observational study performed over 2 years (2006-2007). Patients who developed MRPA nosocomial pneumonia and were treated with aerosolized colistin were included. The criteria used to assess if treatment was successful were extubation and ICU mortality rates.

Results. We report 32 patients of whom 12 were women and 20 men. The mean age was $48 \pm 19$ years. All patients were receiving mechanical ventilation. The mean length of ventilation was $22 \pm 5.5$ days. The bronchial sampling technique used was broncho-alveolar lavage. The mean delay of infection (duration between intubation and pneumonia diagnosis) was $7 \pm$ 2 days. Isolated MRPA was susceptible only to colistin. The treatment was aerosolized colistin for all patients (4 MUI/day). A positive blood culture $(n=5)$ was a prerequisite for administering colistin intravenously (4 MUI/day). Any potential toxicity was observed. The mean delay of extubation after starting treatment was 10 days. Sterile samples were obtained on average by the eighth day. No deaths were recorded.

Conclusion. It seems that aerosolized colistin is an important alternative to treat MRPA nosocomial pneumonia in ICU. Our results need further confirmation by other multicentre studies.
\end{abstract}

Keywords: multi-resistant Pseudomonas aeruginosa, colistin, nebulization, ICU

\section{Introduction}

Nosocomial pneumonia due to multiresistant Gram-negative bacteria, such as Pseudomonas aeruginosa and Acinetobacter baumannii strains, is among the most serious complications that occur in the intensive care unit (ICU). Increasing rates of resistance among Gram-negative bacteria to most classes of antimicrobial agents have frequently led to clinical failure of current therapies. The lack of development of new antibiotics in the treatment of multi-resistant Gram-negative infections has stimulated a renewal of interest in the use of colistin. (1) We report our experience with aerosolized colistin in the treatment of MRPA nosocomial pneumonia (NP).

\section{Patients and methods}

We report a prospective, observational study, conducted over 2 years (20062007) in the Medical Intensive Care Unit in Ibn Rochd University Hospital, Casablanca (Morocco). We included critically ill patients with ventilator-associated pneumonia (VAP) caused by MRPA and treated by aerosolized colistin. Criteria of successful treatment used were extubation and mortality rates. The clinical prerequisites for diagnosis of VAP were as follows: the presence of at least two episodes of fever ( $>38.3$ $\left.{ }^{\circ} \mathrm{C}\right)$, leucocytosis or leucopenia, purulent bronchial secretions, plus a new or persistent infiltrate on chest radiography. The bronchial sampling technique was bronchoalveolar lavage (BAL) with a semi-quantitative method. All strains of $P$. aeruginosa were resistant to all antibiotics apart from colistin. Polymicrobial infection was a criterion for exclusion. All patients were treated with aerosolized colistin $\left(1 \times 10^{6} \mathrm{IU}\right.$ three times a day) for 15 days. Colistin was administered intravenously in patients with a positive blood culture $\left(1 \times 10^{6} \mathrm{IU}\right.$ four times a day) for 21 days. The use of $\beta_{2}$ agonists was necessary if a patient developed bronchospasm. To define acute renal failure, we used the RIFLE classification. (2) Susceptibility testing was performed using the disk diffusion method. The bacteriological monitoring (BAL and blood culture) was performed 
every two days until the $10^{\text {th }}$ day. The definition of a positive outcome of VAP was based on clinical (fever defervescence, resolution or partial resolution of presenting symptoms and signs of pneumonia, decrease in suctioning requirements), radiological (decrease or disappearance of presenting findings on chest X-ray) and laboratory findings (improvement in arterial blood gases or normalization of white blood cell count and $\mathrm{C}$ reactive protein).

\section{Results}

We report 32 patients (12 women and 20 men). The mean age was $48 \pm$ 19 years. All patients were receiving mechanical ventilation (mean length was $22 \pm 5.5$ days). Bronchial sampling technique was broncho-alveolar lavage. The mean delay of infection was $7 \pm 2$ days. The isolated MRPA was susceptible only to colistin. The treatment was based on aerosolized colistin for all patients $\left(3 \times 10^{6} \mathrm{lU} /\right.$ day). Intravenous administration (4 x $10^{6} \mathrm{IU} /$ day) was commenced in the event of a positive blood culture $(n=5)$. No neurological, pulmonary or renal adverse effects to colistin were noted. The level of muscle weakness was not assessed. The mean delay of extubation after starting treat- ment was 10 days. The evolution was favorable for all patients, with sterile bronchial secretions by the $10^{\text {th }}$ day. Blood cultures were negative after 72 hours. No patients died. The duration of stay in ICU was $31 \pm 3$ days.

\section{Discussion}

Colistin and polymyxin E are old antibiotics. Colistin was almost abandoned for many years. This medication was reintroduced into clinical practice just a few years ago. This resulted mainly from increased resistance rates among Gram-negative bacteria especially in the ICU, and the absence of new, effective and alternative therapeutic options. $(3,4)$ The inhalation of colistin in the management of nosocomial pneumonia due to Gram-negative bacteria is not recent. In 1963, Pino (5) used aerosolized colistin in patients with pulmonary infection. A few years later, Marschke and Sarauw (6) reported two cases of pneumonia due to $P$. aeruginosa strains in patients with underlying bronchiectasis and chronic bronchitis. Polymyxin B was administered by inhalation. Actually, Michalopoulos and Motaouakkil $(1,3,7)$ concluded that aerosolized colistin should be a beneficent adjunctive therapy in the manage- ment of nosocomial pneumonia due to multi-resistant Gram-negative bacteria. In our series, none of the patients experienced chest tightness, bronchoconstriction, or apnea. All patients received concurrent treatment with an inhaled $\beta_{2}$ agonist. Among patients who received intravenous colistin, none experienced deterioration of kidney function during the period of treatment. However, our study presents some limitations because of the small case series and the lack of control groups who are only receiving treatment intravenously. Colistin retains significant in vitro activity against multi-resistant $P$. aeruginosa. It possesses an acceptable safety profile and should be considered as a therapeutic option in critically ill patients with infections caused by multidrug-resistant (MDR) Gramnegative bacilli. Aerosolized colistin may deserve further consideration as a salvage therapy for patients with refractory pulmonary infections. (8)

\section{Conclusion}

It seems that aerosolized colistin is an important alternative in the treatment of MRPA nosocomial pneumonia in ICU. Our results need confirmation by other multicentre studies.

\section{REFERENCES}

1. Michalopoulos A, Kasiakou SK, Mastora Z, Rellos K, Kapaskelis AM, Falagas ME. Aerosolized colistin for the treatment of nosocomial pneumonia due to multidrug-resistant Gram-negative bacteria in patients without cystic fibrosis. Crit Care 2005,9:53-59.

2. Bellomo R, Ronco C, Kellum JA, Mehta RL, Paul Palevsky. Acute renal failure - definition, outcome measures, animal models, fluid therapy and information technology needs: the Second International Consensus Conference of the Acute Dialysis Quality Initiative (ADQI) Group. Crit Care 2004:8:204-12.

3. Michalopoulos AS, Tsiodras S, Rellos K, Mentzelpoulos S, Falagas ME. Colistin treatment in patients with ICU acquired infections caused by multiresistant gram-negative bacteria: the renaissance of an old antibiotic. Clin Microbiol Infect 2005;11:115-21.

4. Linden PK, Kusne S, Coley K, Fontes P, Kramer DJ, Paterson D. Use of parenteral colistin for the treatment of serious infection due to antimicrobial-resistant Pseudomonas aeruginosa. Clin Infect Dis 2003; 37:154-60.

5. Pino G, Conterno G, Colongo PG. Clinical observations on the activity of aerosol colimycin and of endobronchial instillations of colimycin in patients with pulmonary suppurations. Minerva Med 1963;54:2117-22.

6. Marschke G, Sarauw A. Polymyxin inhalation therapeutic hazard. Ann Intern Med 1971;74:144-5.

7. Motaouakkil S, Charra B, Hachimi A, Nejmi H, Benslama A, Elmdaghri N, et al. Colistin and rifampicin in the treatment of nosocomial infections from multiresistant Acinetobacter baumannii. J Infect 2006;53:274-8.

8. Mastoraki A, Douka E, Kriaras I, Stravopodis G, Manoli H, Geroulanos S. Pseudomonas aeruginosa susceptible only to colistin in Intensive Care Unit Patients. Surg infect 2008;9:153-60. 